\title{
TIMING OBSERVATIONS OF THE SMC BINARY PSR J0045-7319
}

V. M. KASPI

IPAC/Caltech/Jet Propulsion Laboratory

Pasadena, CA 91125, U.S.A.

R. N. MANCHESTER AND M. BAILES

ATNF/CSIRO

Epping, Australia

AND

J. F. BELL

ANU/Mount Stromlo and Siding Spring Observatories

Canberra, Australia

\begin{abstract}
We describe radio timing observations of the binary pulsar PSR J0045-7319 made over the past 3.3 yr. We show that a simple timing model involving a standard Keplerian orbit describes the data well; however, significant low-level systematic residuals never before seen in other binary pulsars remain. We consider various possible origins of the residuals.
\end{abstract}

\section{Introduction}

PSR J0045-7319 (PSR B0042-73) was discovered in a systematic search of the Magellanic Clouds for radio pulsars (McConnell et al. 1991). The pulsar's association with the SMC is evidenced by its high dispersion measure of $105 \mathrm{pc} \mathrm{cm}^{-3}$, since models of the galactic electron distribution account for no more than $\sim 25 \mathrm{pc} \mathrm{cm}^{-3}$ along that line of sight (Taylor \& Cordes 1993). PSR J0045-7319 is the only known radio pulsar in the SMC, and is the most distant pulsar known.

Radio timing observations of the pulsar by Kaspi et al. (1994) have shown that it is in a 51 day binary orbit with a companion having a minimum mass of $4 \mathrm{M}_{\odot}$. Although the pulsar is a faint source (its flux density at $430 \mathrm{MHz}$ is only $\sim 1 \mathrm{mJy}$ ), its large distance makes it easily the most

J. van Paradijs et al., (eds.), Compact Stars in Binaries, 271-277.

(C) 1996 IAU. Printed in the Netherlands.

(c) International Astronomical Union - Provided by the NASA Astrophysics Data System 


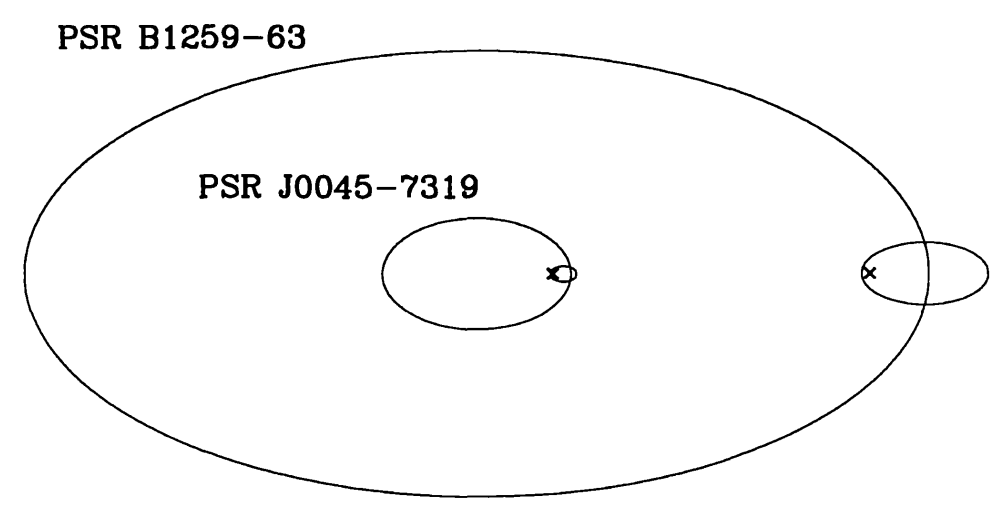

Figure 1. Relative sizes of the PSRs B1259-63 and J0045-7319 orbits, assuming $10 \mathrm{M}_{\odot}$ companions in both cases. The orbits of both binary components are shown. Crosses mark locations of the binary centers of mass.

luminous binary radio pulsar known. Optical observations in the direction of the pulsar have revealed a $\mathrm{V}=16 \mathrm{mag} \mathrm{B} 1$ star at the timing position. The probability of chance alignment being small, we conclude that the PSR J0045-7319 binary system is the second in a new class of binary pulsars having massive, non-degenerate companions. The first example of such a system was PSR B1259-63, reported by Johnston et al. (1992) and also discussed in this volume by Johnston. A schematic drawing of the relative sizes of the two orbits is given in Fig. 1, where we have assumed $10 \mathrm{M}_{\odot}$ companions in both systems, consistent with estimates from optical photometry and spectroscopy.

\section{Timing Properties}

We have made timing observations of PSR J0045-7319 for 3.3 yr at the 64$\mathrm{m}$ Parkes radio telescope. We obtained a total of 155 pulse arrival times from February 1991 through June 1994. Most observations were made at $430 \mathrm{MHz}$; however, a few were made at 660 and $1520 \mathrm{MHz}$. The standard pulse timing analysis was done using the TEMPO software package (Taylor \& Weisberg 1989). The best-fit timing model including astrometric, spin, and Keplerian orbital parameters is given in Table 1; the post-fit residuals after subtraction of this model are shown in Fig. 2 versus date and in Fig. 3 versus orbital phase.

\section{Discussion}

The residuals in Figs. 2 and 3 clearly show significant deviations from the straightforward spin-down and Keplerian orbit model. We consider here possible origins of the deviations. We note, however, that the deviations, 
TABLE 1. Astrometric, Spin, and Orbital Parameters for PSR J0045-7319 assuming a Keplerian orbit.

\begin{tabular}{ll}
\hline Right ascension, $\alpha(\mathrm{J} 2000)$ & $00^{\mathrm{h}} 45^{\mathrm{m}} 35^{\mathrm{s}} .09 \pm 0^{\mathrm{s}} .08$ \\
Declination, $\delta(\mathrm{J} 2000)$ & $-73^{\circ} 19^{\prime} 03^{\prime \prime} .1 \pm 0^{\prime \prime} .3$ \\
Period $P$ & $0.926275835356(15) \mathrm{s}$ \\
Period Derivative, $\dot{P}$ & $4.486(1) 10^{-15}$ \\
Epoch of period & $\mathrm{MJD} 48964.2000$ \\
Orbital Period, $P_{b}$ & $51.169226(3)$ days \\
Projected semi-major axis, $a_{p} \sin i$ & $174.2540(8) \mathrm{lts}$ \\
Longitude of periastron, $\omega$ & $115^{\circ} .2537(7)$ \\
Eccentricity, $e$ & $0.807995(5)$ \\
Epoch of periastron & MJD 49578.56742(3) \\
r.m.s. timing residual & $15 \mathrm{~ms}$ \\
\hline
\end{tabular}

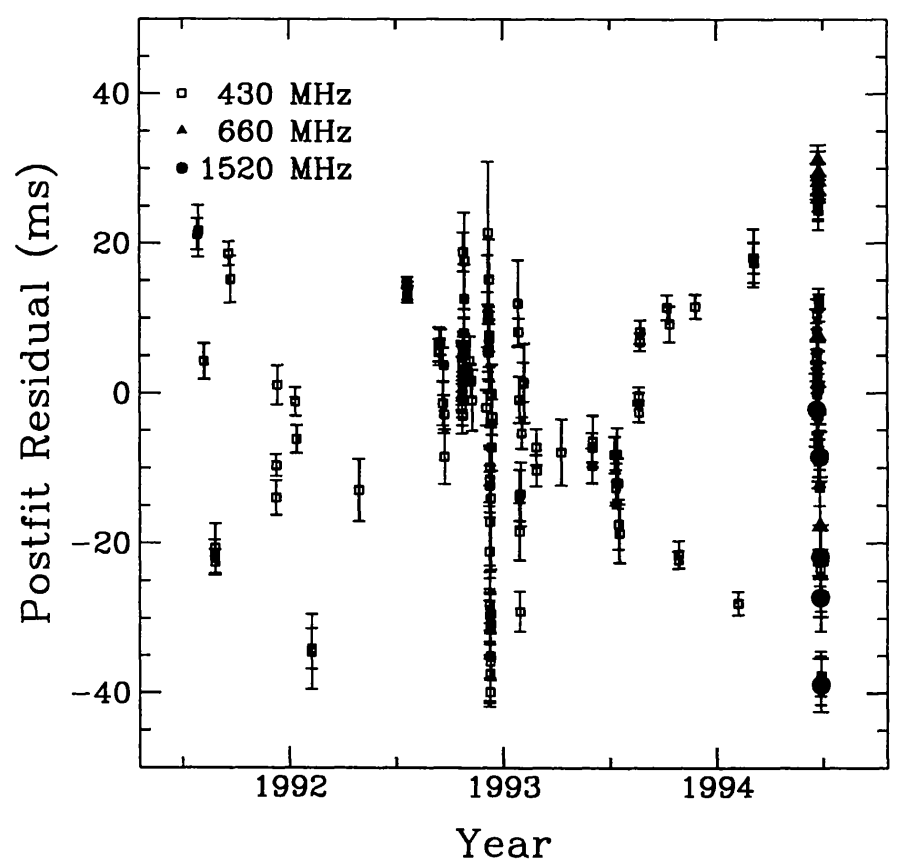

Figure 2. Residuals versus time using the model given in Table 1.

though significant, are low-level, with maximum excursions under $5 \%$ of the pulse period. Thus, we are effectively probing the details of the dynamics of this main-sequence star binary system with unprecedented precision. 


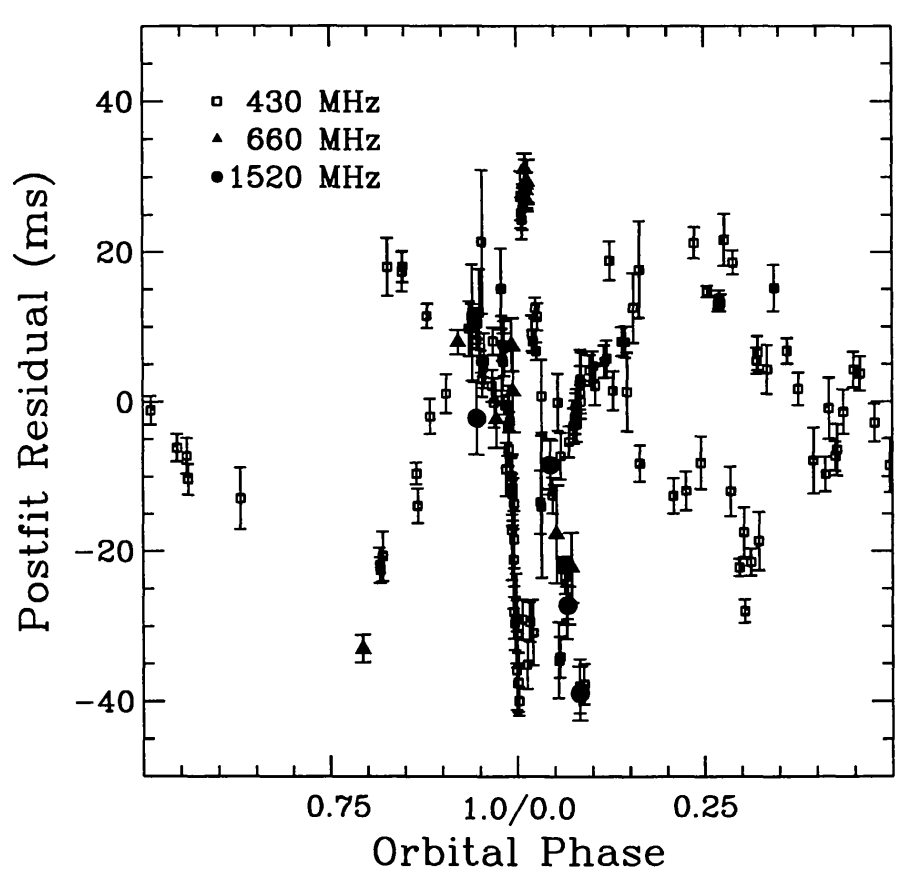

Figure 3. Residuals versus orbital phase using the model given in Table 1.

\subsection{TIMING NOISE?}

Deviations from simple spin down models amounting to significant fractions of the pulsar period are frequently seen in timing data from isolated pulsars (e.g., Arzoumanian et al. 1994, Kaspi 1994), and are usually ascribed to rotational instabilities inherent to the pulsar itself. The timing residuals for PSR J0045-7319, however, do not share the salient characteristics of standard "timing noise". Timing noise typically has a "red" spectrum, that is, has more power at long time scales, with spectral indexes ranging from -2 to -6 (Groth 1975). By contrast, the residuals for PSR J0045-7319 as shown in Fig. 2 show no sign of long-term modulation. Further, timing noise is a phenomenon most common to young pulsars having characteristic ages $\simeq 10^{5} \mathrm{yr}$, and has been shown to be correlated with $\dot{P}$ (Cordes \& Downs 1985). Here, however, the pulsar's characteristic age is $310^{6} \mathrm{yr}$; its $\dot{P}$ is correspondingly small. Thus, standard pulsar timing noise seems an unlikely source of the residuals.

\subsection{ADDITIONAL ORBITAL COMPANION?}

Unexplained deviations of pulse arrival times from simple spin-down models have also been used as evidence for an unseen orbital companion (Backer, Foster \& Sallmen 1993; Thorsett, Arzoumanian \& Taylor 1993). From Figs. 2 and 3, it is clear that any additional orbital companion must have 
an orbital period much shorter than the length of the data span. However, a Fourier transform of the residuals reveals no obvious periodicities.

\subsection{APSIDAL ADVANCE?}

Inspection of the residuals as a function of orbital phase (Fig. 3) reveals no obvious trends such as might be expected for a secular variation of a single Keplerian parameter, such as the longitude of periastron. Apsidal advance at the level of a few hundredths of a degree per year is expected in this system because of the tidal deformation of the companion near periastron, as well as from rotational deformation of the companion and general relativistic effects (Kaspi et al. 1994). Such an effect would be at a similar level to the observed deviations from the Keplerian orbit, but would have an obvious signature.

\subsection{DISPERSION MEASURE VARIATIONS?}

In Fig. 4 we show two well-sampled residual time series, both taken around periastron. The upper panel shows the residuals as a function of orbital phase (which is also time since we are looking at a single orbit) at periastron on MJD 48964. All data at this epoch were taken at $430 \mathrm{MHz}$. The obvious systematic trend could be due to an orbital-phase-dependent variation in the line-of-sight integrated electron density, the dispersion measure, due to an ionized wind from the B star. A variation in the integrated electron density will result in time delays or advances of the pulsar signal because radio waves are dispersed by free electrons according to the standard $1 / f^{2}$ plasma law, where $f$ is the observing frequency.

To test this hypothesis, we repeated the detailed periastron observation 11 orbits later, this time, observing at three different radio frequencies, 430, 660 , and $1520 \mathrm{MHz}$. The results of these June 1994 observations are shown in the lower panel of Fig. 4. It is clear that arrival times at all frequencies are consistent within the measurement uncertainties, which argues strongly against the stellar wind as an origin of the timing residuals. These results can be used to set an interesting and uniquely direct limit on the B star mass loss; the implied upper limit on $\dot{M}$ is $\sim 10^{-11} \mathrm{M}_{\odot} \mathrm{yr}^{-1}$ for a wind velocity of $1000 \mathrm{~km} \mathrm{~s}^{-1}$.

\subsection{TIDAL INTERACTION?}

In the absence of alternatives, a remaining possibility for the origin of the PSR J0045-7319 residuals lies in a gravitational interaction between the components that results in a deformation of the companion's field. However such possibilities have been considered in detail in recent work by Kumar, 


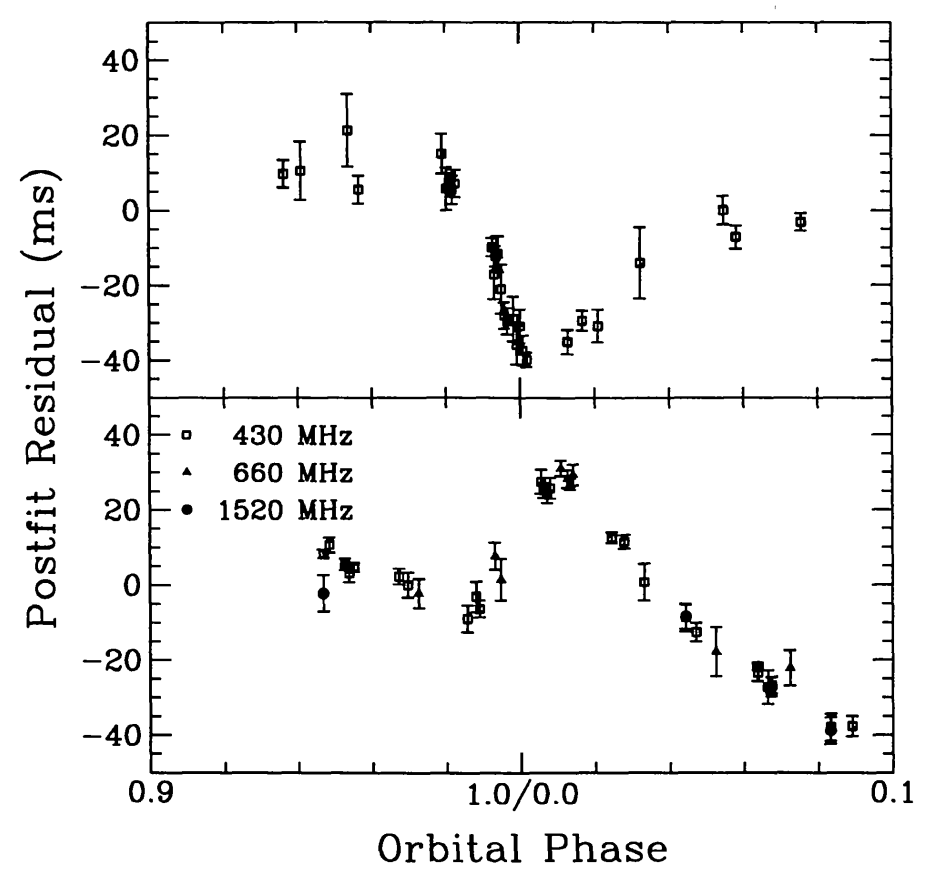

Figure 4. Residuals versus time at two periastron epochs. The upper panel is around periastron on MJD 48964, while the lower panel is around periastron of MJD 49527, 11 orbits later. Periastron is at orbital phase $0.0 / 1.0$, and the timing model used is given in Table 1. The duration of the orbital phase interval shown in these figures is $\mathbf{1 0}$ days.

Ao \& Quataert (1994), who conclude that apart from apsidal advance, no deviations from a Keplerian orbit should be detectable.

\section{Conclusions}

Timing observations of the PSR J0045-7319 binary system over a $3.3 \mathrm{yr}$ period have revealed systematic deviations of pulse arrival times from a simple Keplerian orbit. We have considered several possible origins of these deviations, however their origin remains unclear. In the process, however, we have set a limit on mass loss from the B star that is low, but not incompatible with other highly model-dependent determinations of B star mass loss rates (Snow 1982). The systematic variation in the residuals near periastron suggests they are due to a dynamical interaction between the neutron star and B star, rather than to the neutron star itself. Thus, the above timing results provide strong evidence against a proposed black hole/pulsar model of the PSR J0045-7319 system (Lipunov, Postnov \& Prokhorov 1994) (independent of the persuasive optical observations) since a neutron star/black hole binary should be an extremely clean system, similar to the well-studied neutron star/neutron star binaries (e.g., Taylor \& Weisberg 1989). 


\section{References}

Arzoumanian, Z. et al. 1994, ApJ 422, 671

Backer, D.C., Foster, R.S. \& Sallmen, S. 1993, Nat 365, 817

Cordes, J.M. \& Downs, G.S. 1985, ApJS 59, 343

Groth, E.J. 1975, ApJS 29, 443

Johnston, S. et al. 1992, ApJ 387, L37

Kaspi, V.M. 1994, $\mathrm{PhD}$ thesis, Princeton University

Kaspi, V.M. et al. 1994, ApJ 423, L43

Kumar, P., Ao, C.O. \& Quataert, E.J. 1994, (preprint)

Lipunov, V.M., Postnov, K.A. \& Prokhorov, M.E. 1994, ApJ (in press)

McConnell, D. et al. 1991, MNRAS 249, 654

Snow, T.P. 1982, ApJ 253, L39

Taylor, J.H. \& Cordes, J.M. 1993, ApJ 411, 674

Taylor, J.H. \& Weisberg, J.M. 1989, ApJ 345, 434

Thorsett, S.E., Arzoumanian, Z. \& Taylor, J.H. 1993, ApJ 412, 33

\section{Discussion}

V.M. Lipunov: I think that the B star is not connected to a binary pulsar, but a black hole (see Lipunov, Postnov \& Prokhorov, 1994, ApJ, in press). The main arguments are the absence of a radio eclipse and the absence of a high space (radial) velocity of the B star. 